\title{
Influence of $\gamma$-Irradiation on the Structural Properties of Indium Monoselenide Crystals
}

\author{
F. A. Al-Mufadi \\ College of Engineering \\ Alqassim University \\ Saudi Arabia \\ almufadi@qec.edu.sa
}

\author{
A. El-Taher \\ Physics Dept, Faculty of Science, \\ Al-Azher University, Assuit Branch, \\ Egypt \\ atef_eltaher@hotmail.com
}

\author{
G. A. Gamal \\ Physics Dept, South Valley University, \\ Egypt and \\ College of Engineering, Alqassim \\ University, Saudi Arabia \\ profdrgamal@qec.edu.sa
}

\begin{abstract}
Indium monoselenide (InSe) which is a layered semiconductor whose energy gap is $1.24 \mathrm{eV}$ has received attention because of its potential applications in optoelectronic devices. In the present work n-type InSe crystals were grown by a special modification of the vertical Bridgman technique. X-ray Diffraction (XRD) patterns showed that all samples were single phased InSe. The crystals were irradiated with 100 - 500 KGy to reveal the influence of irradiation on the structural properties of the samples. Detailed structural analysis was done by Scherrerr's procedures, $x$-ray peak broadening and Williamson-Hall plot methods to evaluate the crystalline sizes and lattice strain. Also from the relations between the irradiation dose and the deformation of the lattice parameters we could investigate and discuss the mutation of the dislocation density, grain size, microstrain and crystallinity of the grown crystals. Striking results are found due to irradiation in the present study for the first time.
\end{abstract}

Keywords-Crystallite Size; Dislocation; InSe Crystals; Micro strain; Radiation Effects; XRD Analysis

\section{INTRODUCTION}

Binary chalcogenide compounds of III-VI semiconductor like InSe, InTe, and InS have experienced great developments in the last few decades. Among them InSe is the most studied both experimentally and theoretically. InSe is a layered semiconductor whose energy gap is $1.24 \mathrm{eV}[1,2]$ and has ntype conductivity [3]. $\operatorname{lnSe}$ is formed by "bidimensional" layers stacked with Se-ln-ln-Se sequence. Each layer is bounded to its neighbor by Van der Waals bonds. So cleavage occurs perpendicularly to the c- axis. Literature survey revealed that it is one of the most suitable compounds of semiconductors for optoelectronic and photovoltaic applications [4]. This semiconductor has been used to design technologically many devices, such as solar energy converters, infrared detectors, optical fibers, solar elements and other photoelectric sensors [5]. It has been reported that the photoconductive response is mostly in the visible region [6]. Also from literatures one can conclude that the results concerning the structure and physical properties of $\ln S e$ are often contradictory and it depends on the perfection of the grown crystals [7].
It is an established fact that these discrepancies are the consequence of the poor quality of the grown crystals [8]. It has been found that the efficiency of InSe solid state devices is limited by the defects which are created during crystal growth [9]. Also similarly to other crystals, the preparation method plays a very important role with regard to the structural properties. Some researchers intend to add excess of In because it enables the growth of easily cleavable layered crystals. This is why we introduced a modification to the vertical Bridgman technique in order to obtain high quality crystals because of these modifications. In fact a perfect crystal would extend in all directions to infinity, so no crystals are perfect due to their finite size of the products. This deviation from perfect crystallinity leads to a broadening of the x-ray diffraction peaks.

The two main properties extracted from peak width analysis are (a) crystallite size and (b) lattice strain. Crystallite size is a measure of the size of a coherently diffracting domain [10]. So the current research is an attempt to find the effect of irradiation on the quality of the grown crystals. Another reason encouraged us to undertake such study where InSe crystals may suffer from radiation damage as pre-mentioned above during the review applications or even it is a candidate under development for the next generation of high-energy physics experiments. Generally since the main goal of the present study is to estimate the structural properties of the crystals and their relations under the influence of the $\gamma$ - irradiation doses, we did apply x-ray profile analysis as a simple and powerful tool for this purpose [11].

\section{EXPERIMENTAL TECHNIQUE}

\section{A. Crystal Growth}

Stoichiometric proportions of indium and selenium was used to produce InSe crystals. The charge elements were: In ( $6 \mathrm{~N}$ pure), and $\mathrm{Se}(5 \mathrm{~N}$ pure) as obtained from Aldrich. The starting materials were placed in a sealed evacuated silica tube ( $\sim 10^{-6}$ Torr). For growing single crystals of InSe a new method, which is illustrated in Figure1, was used. This technique is very simple but it enabled us to obtain crystals of high quality. This 
is because of the absence of motor vibrations. Details of this technique have already been published [12]. Among the advantages of this method is that the growth rate can be controlled easily. The ampoule is moved downward in the thermal gradient of $40^{\circ} \mathrm{C} / \mathrm{cm}$, between the temperatures of 750 and $250^{\circ} \mathrm{C}$, with a speed of $3 \mathrm{~mm} / \mathrm{h}$. Prior to the growth process, the In and Se charge was slowly heated up to $750^{\circ} \mathrm{C}$ and maintained at this temperature for 12 hours to ensure melting and complete the reaction. During the growth process the tube with its charge were held at $660^{\circ} \mathrm{C}$ (according to the phase diagram [13]). The grown crystals were identified by means of x-ray diffraction since it is an established fact that the $\mathrm{x}$-ray diffraction is a good tool for verifying crystals. Also the lattice parameters, grain size, strain and dislocation density of a given material can be determined by using $\mathrm{x}$-ray investigations [11]. The conformation of the product crystal showed that it is single phase with a monoclinic structure $(\mathrm{C} 2 / \mathrm{m}(12))$ and the unit cell parameters obtained are the same as reported earlier [13].The samples were irradiated with $\gamma$-rays obtained from cells available at King Saud University. The average dose rate from the cell was $1 \mathrm{~Gy} / \mathrm{S}$. The doses were adjusted to be 100 KGy (for about 1day), $300 \mathrm{KGy}$ (for about 4 days) and $500 \mathrm{KGy}$ (for about 6 days).

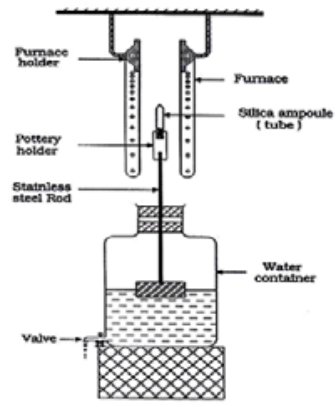

Fig. 1. Method for growth of single crystals of Indium monoselenide

\section{B. X-Ray Diffraction Technique}

The $\mathrm{x}$-ray diffractograms were measured with scan speed 2 $\mathrm{deg} / \mathrm{min}$ (continuous scan mode) at ambient temperature with a Goniometer type Ultima IV. The instrument is equipped with a copper anode generating $\mathrm{Ni}$ filtered $\mathrm{CuK} \alpha$ radiation $(\lambda=1.5406$ $\mathrm{A}^{\mathrm{o}}, 40 \mathrm{kV}, 40 \mathrm{~mA}$, backmonochromator). The equipment was used in a $\theta-2 \theta$ geometry in the range between 10 and $80^{\circ}$ with a divergence slit of $2 / 3 \mathrm{deg}$. In the present work, we will show how we can utilize the $\mathrm{x}$-ray pattern for determination of many important parameters. It must be mentioned that background subtraction and $\mathrm{Ka} 2$ elimination were done before the peak search work.

\section{RESULTS \& DISCUSSION}

The measurement of structural parameters by means of $\mathrm{x}$ ray diffraction is of great interest from both experimental and theoretical point of view. The direction of the reflection beams is determined entirely by geometry of the lattice which in turn is governed by the orientation and spacing of the crystalline planes. If for crystal of symmetry the given size of the structure cell $\mathrm{a}, \mathrm{b}$ and $\mathrm{c}$, the angle at which the beam diffraction from the crystal plane (hkl) can easily be calculated from the interplanar spacing relationships.

\section{A. XRD analysis}

The measurement of structural parameters by means of $\mathrm{X}$ ray diffraction has therefore of many advantages over the other techniques. For example it does not require time for sample preparation (tinning) and image analyzing. Utilization of the $\mathrm{x}$ ray diffraction has been done to reveal the influence of irradiation on the crystal structure of the grown InSe crystals. Figure 2 shows the powder diffractogram of the InSe sample. In this figure, we can see strong Bragg peaks of x-ray diffraction pattern which indicate that the sample is crystalline. No other or foreign peaks were detected. The intense peaks in the patterns prove the high crystallinity of the products. The relative intensities of the reflection peaks in all patterns agree well with the XRD pattern of bulk InSe. Also the effect of irradiation on InSe structure is present in XRD charts. This can be observed as a change in the peak intensities. The variations of the intensities of the main peaks is due to that localized variations in intensity within any individual diffracted spot arise from structural non-uniformity in the lattice planes causing the spot, and this forms the basis for the $\mathrm{x}$-ray topographic technique.

This topographic contrast arises from differences in the intensity of the diffracted beam as a function of position inside the crystal. So this change, simply, is a function of crystallinity. On the other hand, with increasing irradiation doses from 100 to 300 and 300 to $500 \mathrm{KGy}$, the crystallinity of the products was changed. In this work, it is desired to establish the extent to which the irradiation treatment plays a significant role in improvement of the crystal quality. 300 KGy shows the preferable dose that can be used in this respect where at $500 \mathrm{KGy}$ the peak intensity is no longer high. Now if the crystallite is strained then the $d$ spacing will be changed; a compressive stress would make the d-spacing smaller (and a tensile stress would make the $d$ spacing larger), say reducing a given spacing $d$ to $d-\delta d$. Then by Bragg's Law the position of the peak will increase from $2 \theta$ to $2(\theta+\delta \theta)$. If every crystallite in the sample was strained (compressed) by the same amount it would result in a peak shift from $(2 \theta)$ to $2(\theta+\delta \theta)$. Based on the above facts and regarding to Figure 2 we concluded the following:

1. All the three patterns prove that the crystal, understudy, is confirmed as InSe monoclinic if compared to data and the d spaces of [13].

2. Under the influence of irradiation the peaks shift upward. Since the peak height depends on the crystal quality, so it is easy to conclude that irradiation improves the crystallinity and hence the crystal quality.

3. In spite of the observable broadening, the peak positions are the same for the different irradiation doses. This indicates that the compressive stress is excluded.

4. Irradiation improves the crystallinity and the crystal quality but until a certain limit. This is concluded where peaks started to decrease above $300 \mathrm{KGy}$. 


\section{B. Determination of Crystal Lattice Parameters}

The direction of the reflection beams is determined entirely by geometry of the lattice which in turn governed by the orientation and spacing of the crystalline planes. If for crystal of symmetry the given size of the structure cell a, b and c, the angle at which the beam diffraction from the crystal plane (hkl) can easily be calculated from the inter-planar spacing relationships. We did so and the obtained data were used to identify the product crystal. Moreover with the aid of Bragg's law: $\mathrm{n} \lambda=2 \mathrm{~d} \sin \theta$ we calculated, by computational treatments, structure cell $\mathrm{a}, \mathrm{b}$ and $\mathrm{c}$ substantially free from experimental error by using the extrapolation function $\mathrm{F}(\theta)$.

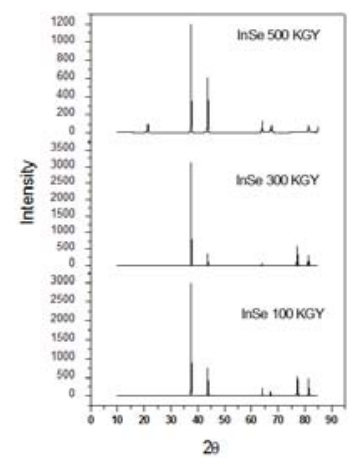

Fig. 2. X-ray charts of InSe crystals at 100, 300 and $500 \mathrm{KGy}$

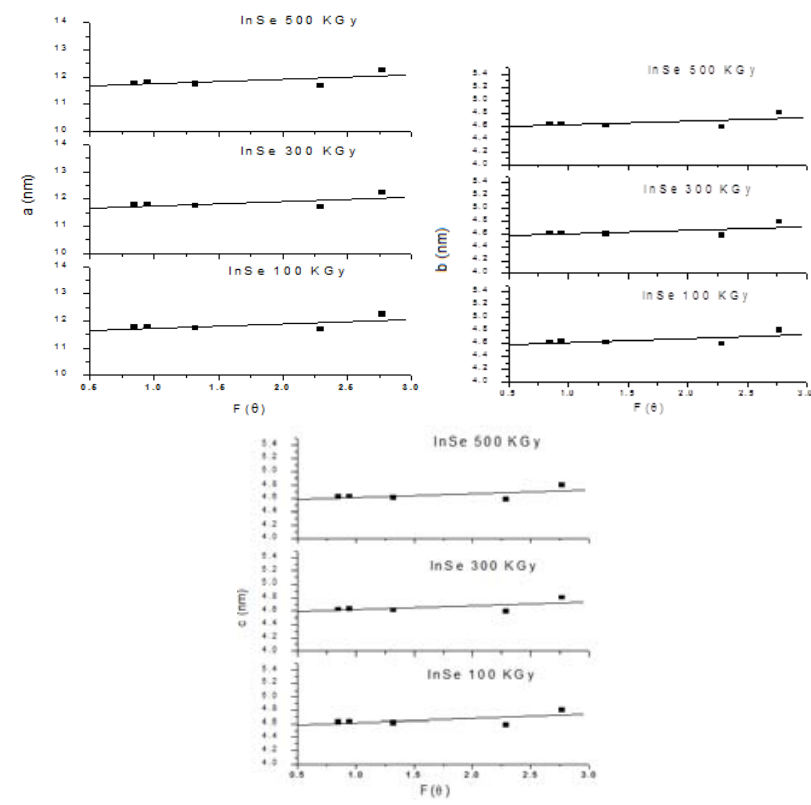

Fig. 3. Relation between the lattice parameters $a, b$ and $c$ and the extrapolation function F ( $\theta$ ) for InSe crystals at 100, 300 and $500 \mathrm{Kgy}$

From the values of $d$-spaces, for different reflection planes, we calculated the lattice parameters of the monoclinic InSe according to the following equation [14]: $\frac{1}{\mathrm{~d}_{\mathrm{hkl}}^{2}}=\left[\frac{\mathrm{h}^{2}}{\mathrm{a}^{2}} \sin ^{2} \beta\right]+\left[\frac{\mathrm{k}^{2}}{\mathrm{~b}^{2}}\right]+\left[\frac{1^{2}}{\mathrm{c}^{2}} \sin ^{2} \beta\right]-\left[\frac{(2 \mathrm{~h} 1 \cos \beta)}{\left(\mathrm{a} \operatorname{csin}^{2} \beta\right)}\right]$

Where $\mathrm{d}$ is interplanar spacing, $\mathrm{h}, \mathrm{k}$ and $\mathrm{l}$ are Miller's indices and $\mathrm{a}, \mathrm{b}$ and $\mathrm{c}$ are the lattice parameters. It is observed in Fig.2 that peak broadenings occurred. This is regarded as a result of: (a) crystallite size and (b) lattice strain. For more utilization of Figure 2, we shall digress with a discussion on the width of the main peak in the three cases. The pre-mentioned three cases correspond to the three crystals as irradiated with 100,300 and $500 \mathrm{KGy}$. In order to obtain the lattice parameters $\mathrm{a}, \mathrm{b}$ and $\mathrm{c}$ of the monoclinic InSe substantially free from experimental error, one should plot the apparent values of a, band c respectively against the corresponding values of the famous extrapolation function $\mathrm{F}(\theta)$ which is [15]:

$$
\mathrm{F}(\theta)=\frac{\cos ^{2} \theta}{\sin \theta}+\frac{\cos ^{2} \theta}{\theta}
$$

Figure 3 shows the relation between the lattice parameters $a, b$ and $c$ vs $F(\theta)$. The interception of the extrapolation of straight line with $y$-axis gives the value of the lattice parameters a,b and $\mathrm{c}$ accurately. The results of lattice parameters as estimated from Figure 3 are shown in Table I.

TABLE I. RESULTS OF LATTICE PARAMETERS AS ESTIMATED FROM FIG 3

\begin{tabular}{|c|c|c|c|c|c|c|}
\hline \multirow[t]{2}{*}{ Dose } & \multicolumn{3}{|c|}{ Lattice parameter $A^{0}$} & \multicolumn{3}{|c|}{ Values in [13] $\mathbf{A}^{0}$} \\
\hline & \begin{tabular}{l|l}
$\mathbf{a}$ & \\
\end{tabular} & b & c & $\mathbf{a}$ & b & c \\
\hline (100 kGy) & 11.582 & 4.138 & 4.548 & \multirow{3}{*}{11.74} & \multirow{3}{*}{4.11} & \multirow{3}{*}{4.61} \\
\hline (300 kGy) & 11.581 & 4.137 & 4.548 & & & \\
\hline (500 kGy) & 11.585 & 4.139 & 4.549 & & & \\
\hline
\end{tabular}

The data appeared in Table I and Figure 3 leads to the following comments:

1. Results of a, b and c support that the crystal is monoclinic.

2. Generally all the lattice parameters are influenced by the exposure of irradiation

3. Careful look at the estimated values compared to those reported in [13] leads to conclude that generally a and c values are always less than those of the standards, while $b$ values are higher than the reported standards.

4. From the results and calculations we concluded that the expansion rate of $b$ values is less than the shrinkage rate in $\mathrm{a}$ and $\mathrm{c}$ values. Accordingly, the unit cell volume decreases under the influence of the radiation doses i.e. radiation cause shrinkage. However as concluded from preivous section, tensile stress is excluded.

5. The lowest unit cell volume value was observed at 300 KGy.

6. The fluctuation in the lattice parameters under the different irradiation doses, as observed in Figure 3, may be attributed to the variation occurred in the quality and/or the lattice imperfections of the crystals due to irradiation [16]. 
7. The calculated shrinkage is not the same in all cases of 2 . radiation. The greatest shrinkage is observed at $300 \mathrm{KGy}$, while the least effect is occurred at $500 \mathrm{KGy}$.

\section{Determination of Crystallite Size}

In (3) by substituting the values of $\Gamma$ (the full width at half maximum) and the values of the main peak we can obtain the value $\mathrm{D}_{\text {vol }}$ (crystal size). This quantity is important because it is proportional to $\mathrm{L}_{\mathrm{vol}}$ (the column lengths). The primarily obtained column lengths of an ensemble of particles can be transformed into average grain sizes if all the crystallites in the sample have roughly the same shape [17]. The standard assumption is a spherical shape, then:

$$
\mathrm{D}_{\mathrm{vol}=4 / 3} \mathrm{~L}_{\mathrm{vol}}
$$

This is why we computed $\mathrm{D}_{\mathrm{vol}}$ and $\mathrm{L}_{\mathrm{vol}}$ as shown in Table II. From the Scherer's equation [18]:

$$
\Gamma=\frac{0.94 \lambda}{D_{\mathrm{vol}} \cos \theta}
$$

we can obtain the crystallite size $\left(\mathrm{D}_{\mathrm{vol}}\right)$, and hence $\left(\mathrm{L}_{\mathrm{vol}}\right)$, for the monoclinic InSe crystals at the different conditions understudy .The results are as follows:

TABLE II. RESULTS FOR MONOCLINIC INSE CRYSTALS AT DIFFERENT CONDITIONS

\begin{tabular}{|c|c|c|}
\hline Dose & $\mathbf{L}_{\text {vol }}$ & D $_{\text {vol }}$ \\
\hline $100 \mathrm{KGy}$ & 38.534 & 51.38 \\
\hline $300 \mathrm{KGy}$ & 51.120 & 68.16 \\
\hline $500 \mathrm{KGy}$ & 37.571 & 50.10 \\
\hline
\end{tabular}

In spite of Scherer's formula beina an approximation, it is observed that the values of $\mathrm{D}_{\mathrm{vol}}$ and hence $\mathrm{L}_{\mathrm{vo}}$ are inversely proportional to the unit cell volumes that estimated in previous section. Also the Williamson-Hall equation can be given as [19]:

$$
\Gamma \cos \theta=\frac{0.94 \lambda}{\mathrm{D}_{\mathrm{vol}}}+4 \varepsilon \sin \theta
$$

Where $\Gamma$ is the full width at half-maximum of $x$-ray peak at diffraction angel $\theta$. We utilized Williamson- Hall relation because it takes into account that the total width of the x-ray diffraction peak is due to both size and strain effects. For the separation, the different dependence is helpful: the size broadening is proportional to $\cos ^{-1} \theta$ and the strain broadening is proportional to $\tan \theta$. Accordingly from plotting the relation between $\Gamma \cos \theta$ and $\sin \theta$, we can get from the intercept the value of $\mathrm{D}_{\mathrm{vol}}$ and from the slope the value of the micro- strain. This is done in Figure 4 regarding to Williamson-Hall relation. From the figure and the results we can conclude the listed data as shown in Table III.

As for the obtained results in Table III, the following comments can be made:

1. Both $\mathrm{D}_{\mathrm{vol}}$ and $\mathrm{L}_{\mathrm{vol}}$ are influenced by the irradiation.
2. Both $\mathrm{D}_{\mathrm{vol}}$ and $\mathrm{L}_{\mathrm{vol}}$ have the highest values when the crystals are irradiate to $100 \mathrm{KGy}$ while the lowest are corresponding to300 KGy.

3. The above result is acceptable if we consider the peak broadening appeared in Figure 2. Again XRD peaks are broadened by small crystallite size and lattice distortion caused by lattice dislocations.

4. Generally the values of crystallite size calculated by Scherrer's method are less than that obtained by Williamson-Hall method. This difference is attributed to the strain value and confirms that the role of strain is important.

5. This difference should be considered in the calculation of crystalline size. Thus, calculations by using the Scherrer's method without considering strain may yield inaccurate results.

TABLE III. RESULTS

\begin{tabular}{|c|c|c|c|c|}
\hline Dose & $\begin{array}{c}\mathbf{L}_{\mathbf{v o l}} \\
(\mathbf{n m})\end{array}$ & $\mathbf{D}_{\text {vol }}(\mathbf{n m})$ & Microstrain & $\begin{array}{c}\text { Dislocation } \\
\left.\text { density } \mathbf{( m}^{\mathbf{2}}\right)\end{array}$ \\
\hline $100 \mathrm{KGy}$ & 186.14 & 248.19 & 0.0017 & $5.57 \times 10^{10}$ \\
\hline $300 \mathrm{KGy}$ & 47.00 & 62.67 & 0.0003 & $1.88 \times 10^{9}$ \\
\hline $500 \mathrm{KGy}$ & 63.49 & 84.66 & 0.0014 & $3.8 \times 10^{10}$ \\
\hline
\end{tabular}

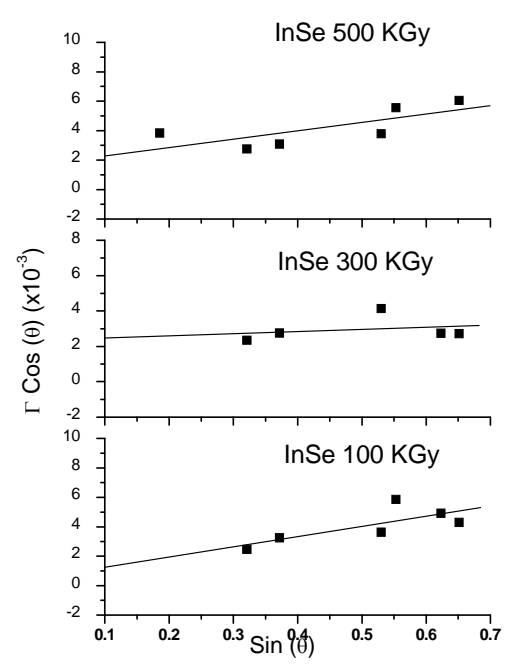

Fig. 4. $\Gamma \cos \theta$ as a function of $\sin \theta$

D Determination of the microstrain and the dislocation density

As it is mentioned Williamson Hall technique takes into account both size and strain effects. The formula which is between $\Gamma \cos \theta$ and $\sin \theta$ is a liner relationship as predicted. From the intercept of the linear we had above the crystal size. The slope of the fitted line provides the micro-strain. The average dislocation density $\mathrm{R}_{\mathrm{e}}$ can be determined from the following equation [20]: 


$$
\varepsilon^{2}=\left(\pi \mathrm{Ab}^{2} / 2\right) \mathrm{R}_{\mathrm{e}} \mathrm{C}
$$

where $\left(b^{2} / 2\right)$ is the Burgers vector, $A$ is a constant defined by the effective outer cut-off radius of dislocations $\mathrm{R}_{\mathrm{e}}$ and $\mathrm{C}$ is the contrast factor.

The results of the microstrain and the dislocation density are listed in Table III. The results reveal the following:

1. Both values of the microstrain and the dislocation density are influenced by the irradiation.

2. Both the microstrain and the dislocation density behave in the same manner.

3. They reach the maximum values at irradiation of $500 \mathrm{KGy}$ while the lowest values are observed at $300 \mathrm{KGy}$.

4. From the dislocation density relations, the average distance between the adjacent dislocations $\mathrm{L}_{\mathrm{c}}$ (as appeared in the previous table) can be checked simply as $\mathrm{L}_{\mathrm{c}}=1 / \sqrt{\rho}$

\section{CONCLUSION}

InSe crystals were grown by a special modification of the vertical Bridgman technique. Via X-ray diffraction examinations it was indicated that the grown samples are crystalline InSe in their monoclinic form. The crystals were irradiated with 100 - $500 \mathrm{KGy}$. The crystallite size and lattice strain were estimated from broadening of XRD peaks by using Scherrer and Williamson-Hall methods. A lot of structural work was done. The basic conclusions are as follows:

1. As the crystals are irradiated: the crystallinity, lattice parameter $\mathrm{a}, \mathrm{b}$ and $\mathrm{c}$, crystal size, the column length, microstrain and dislocation density are influenced by the exposure of irradiation.

2. Irradiation improves the crystallinity and the crystal quality but until a certain limit (300 KGY). X-ray peak positions are the same at the different irradiation doses. This indicates that the compressive stress is excluded.

3. The unit cell volume decreases under the influence of the radiation doses exposure i.e. radiation cause shrinkage. However as concluded from section III.B tensile stress is excluded.

4. Since both values of the microstrain and the dislocation density are influenced by the irradiation, this was utilized to interpret the reason irradiation improves the crystal quality by dislocation reduction which causes unit cell shrinkage.

5. Generally the values of crystallite size calculated by the Scherrer method are lower than the ones obtained by the Williamson-Hall method. This is a minor result if compared to previous work that was already published for other compounds [21]. However similar situations have been reported [22].

\section{REFERENCES}

[1] T. Iishii, "High quality single crystal growth of layered InSe semiconductor by Bridgman technique", Journal of Crystal Growth, Vol 89 , pp. 459-462, 1988

[2] R. Triboulet, C. Levy-Clement, B. Theys, "Growth of InSe single crystals by the travelling heater method", Journal of Crystal Growth,Vol. 79, pp. 984-989, 1986

[3] A. Segura, F. Pomer, A. Cantarero, "Electron scattering mechanisms in n-type indium selenide", Phys. Rev., Vol. B29,pp. 5708, 1984

[4] S. Shigetomi, H. Ohkubo, "Annealing behaviour of optical properties and structure of r.f-sputteredinse films", Thin Solid Films, Vol. 199, pp. $215-222,1991$

[5] J. Hossain, M. D. Julkarnain, K. S. Sharif, K. A. Khan, "Preparation and Properties of Indium Selenide (InSe) Thin Films for Selective Surface Applications", Journal of Physical Science and Application, Vol.1, pp. 37-43, 2011.

[6] A. A. Darwish, M. M. El-Nahass, M. H. Bahlol, "Structural and electrical studies on nanostructured InSe Thin films", Applied Surface Science,Vol. 276, pp. 210-216, 2013

[7] A. Cttevy, A. Kuhn, M. S. Marlin, "Large InSe monocrystals grown from a non-stoichiometric melt", Journal of Crystal Growth, Vol. 38 pp. 118-122, 1977

[8] S. Hirohata, J. S. Moodera, G. P. Berera, "Structural and electrical properties of InSe polycrystalline films and diode fabrication", Thin Solid Films, Vol. 510, pp. 247-250, 2006

[9] C. D. E. Blasi, G. Hicocci, S. Mongelli A.Tepore, F. Zuanni, "Growth and characterization of high quality inse single crystals", Materials Chemistry and Physics, Vol. 9, pp. 55-64, 1983

[10] S. Vives, E. Gaffet, C. Meunier, "X-ray diffraction line profile analysis of iron ball milled powders", Materials Science and Engineering, Vol. 66, No. A3, pp . 229-238, 2004

[11] V. D. Mote, Y. Purushotham, B. N. Dole, "Williamson-Hall analysis in estimation of latticestrain in nanometer-sized $\mathrm{ZnO}$ particles", Journal of Theoretical and Applied Physics, Vol. 6, pp. 1-8, 2012

[12] G. A. Gamal, M. Abou Zied, A. A. Ebnalwaled, "The Electronic Properties of the Red P- type T12S5 Single Crystals", Chinese Physics Letters,Vol. 22, pp. 6, 2005

[13] K. Cenzual, L. M. Gelalo, E. Parthe "Inorganic structure types with revised space group", Acta Cryst., Vol. B47, pp. 433-439, 1991

[14] S. Q. Xiao, A. H. Gleiter, "Nanocrystalline Titanium Aluminide", Acta Metall. Mater., Vol. 42, pp. 2535- 2545, 1994

[15] R. A. Varin, J. Bystrzycki, A. Calka, "Processing and Fabrication of Advanced Materials", Intermetallics, Vol. 7, pp. 917, 1999

[16] C. Castro Ribeiro, I. Gibson, M. Adolfo Barbosa, "The uptake of titanium ions by hydroxyapatite particles - structural changes and possible mechanisms", Biomaterials, Vol. 27, No. 1,pp. 749-176, 2006

[17] C. E. Krill, R. Birringer, "Crystallite size distribution and dislocation structure determined by diffraction profile analysis" Philos. Mag., Vol. 77 , pp. 621,1998

[18] P. Scherrer, "Determining the size and the internal structure of colloidal particles by means of X-rays", Nachr. Ges. Wiss. Göttingen., Vol. 26, pp. 98, 1918

[19] G. K. Williamson, W. H. Hall, "Cold-Worked Iron and Molybdenum", Acta Met.,Vol. 1, pp. 22-28, 1953

[20] M. Wilkens, "The determination of density and distribution of dislocations in deformed single crystals from broadened X-ray diffraction profiles", Physica Status Solidi A, Vol 2, No. 2,pp. 359-370, 1970

[21] G. A. Gamal, F. A. Al-Mufadi, A. H. Said, "Effect of Iron Additives on the Microstructure of Hydroxyapatite", Engineering, Technology \& Applied Science Research,Vol. 3, No. 6, pp. 532-539, 2013

[22] V. D. Mote, Y. Purushotham, B. N. Dole, "Williamson-Hall analysis in estimation of latticestrain in nanometer-sized $\mathrm{ZnO}$ particles", Journal of Theoretical and Applied Physics, Vol. 6, pp. 1-8, 2012 